\title{
Rhabdomyolysis-induced acute kidney injury requiring hemodialysis after a prolonged immobilization at home in 2 morbidly obese women: case reports with literature review
}

Kazuhiro Sakai, Hiroki Omizo, Ryo Togashi, Yuto Hayama, Masaki Ueno, Yoshihiro Tomomitsu, Yoshikazu Nemoto, Shinichiro Asakawa, Michito Nagura, Shigeyuki Arai, Osamu Yamazaki, Yoshifuru Tamura, Shunya Uchida, Shigeru Shibata and Yoshihide Fujigaki ${ }^{*}$ (D)

\begin{abstract}
Background: Rhabdomyolysis may develop into acute kidney injury (AKI), a life-threatening complication. Obese people are at risk for rhabdomyolysis due to prolonged immobilization. However, there are only a few reports of rhabdomyolysis-induced AKI due to prolonged immobilization after falls in morbidly obese people. Myoglobin is a causative compound for rhabdomyolysis-induced AKI, but the lack of treatments targeting its mechanism is a problem.

Case presentation: Two morbidly obese women (body mass index $>40.0 \mathrm{~kg} / \mathrm{m}^{2}$ ) who fell on the floor at home and remained in the same posture for more than $12 \mathrm{~h}$ developed rhabdomyolysis-induced AKI. Both patients received aggressive fluid resuscitation but required hemodialysis because of persistent oliguria. They underwent 11 and 2 intermittent hemodialysis (HD) sessions with a conventional polymethylmethacrylate (PMMA) high-flux dialyzer, respectively, and their renal functions returned to baseline after withdrawal of HD.
\end{abstract}

Conclusions: We should be aware that morbidly obese people are at risk for rhabdomyolysis-induced AKI due to prolonged immobilization, such as after falls. At present, prophylactic renal replacement therapy (RRT) is not recommended for rhabdomyolysis. We need to reevaluate whether RRT using the appropriate membranes to effectively remove myoglobin including the PMMA membrane can improve the renal outcome in patients with rhabdomyolysis-induced AKI.

Keywords: Acute kidney injury, Body mass index, Creatine kinase, High cut-off membrane, Medium cut-off membrane, Morbidly obesity, Myoglobin, Polymethylmethacrylate membrane, Rhabdomyolysis, Renal replacement therapy

\footnotetext{
* Correspondence: fujigaki@med.teikyo-u.ac.jp

Department of Internal Medicine, Teikyo University School of Medicine,

2-11-1 Kaga, Itabashi-ku, Tokyo, Japan
} 


\section{Background}

Rhabdomyolysis is caused by damage to skeletal muscle, resulting in the release of its breakdown products, including myoglobin, into the circulation, and it can lead to acute kidney injury (AKI), a life-threatening complication [1]. Myoglobin is recognized as a causative compound of AKI. Common causes of rhabdomyolysis are trauma (crush syndrome), exertion, muscle hypoxia, genetic defects, infections, body-temperature changes, metabolic/electrolyte disorders, and drugs/toxins [2]. Muscle hypoxia or crush syndrome-like mechanisms can be induced by limb compression by the head or torso during prolonged immobilization [2]. This condition is called pressure-induced rhabdomyolysis [3]. Morbidly obese people are at risk for rhabdomyolysis due to trauma or immobilization after prolonged surgery [4-6]. However, there are a few reports on rhabdomyolysis-induced AKI due to prolonged immobilization after a fall in morbidly obese people.

We describe here two morbidly obese women who showed rhabdomyolysis due to prolonged immobilization after a fall at home. Despite aggressive fluid resuscitation, both patients required hemodialysis (HD) and recovered renal function. It is known that severe AKI is a risk for mortality and the development of chronic kidney disease (CKD) even after full recovery of renal function [7]. No intervention has been demonstrated to prevent AKI in humans. At present, prophylactic renal replacement therapy (RRT) is not recommended in patients with rhabdomyolysis. However, various kinds of dialyzer membranes such as the medium-cut-off (MCO) membrane [8], which can effectively eliminate large to middle-sized molecules, including myoglobin, have been developed and are used in patients with end-stage kidney disease. Thus, we need to reevaluate whether RRT using the appropriate membrane to effectively remove myoglobin could improve renal outcome in patients with rhabdomyolysis-induced AKI.

\section{Case presentation}

\section{Case 1}

A 67-year-old woman fell on her home bathroom floor due to dizziness in the morning and could not move. She was found by her husband $12 \mathrm{~h}$ after the fall. She was lying on her left side, with her left arm underneath her body. Her body and right leg, with the hip joint in a highly flexural position, were trapped between the toilet and the wall. She complained of paralysis of the left upper arm. She was transferred to our hospital. She had a history of hypertension, for which she was taking $4 \mathrm{mg} /$ day of candesartan; hyperlipidemia, for which she was taking $5 \mathrm{mg} /$ day of atorvastatin; and CKD stage G3b, probably due to nephrosclerosis. She had used a wheelchair because of knee joint pain due to osteoarthritis.
Physical examination upon admission showed clouding of consciousness (Glasgow Coma Scale of 14), height of $155 \mathrm{~cm}$, body weight of $101.9 \mathrm{~kg}$, body mass index (BMI) of $42.4 \mathrm{~kg} / \mathrm{m}^{2}$, body temperature of $39.9^{\circ} \mathrm{C}$, blood pressure of $88 / 39 \mathrm{mmHg}$, heart rate of $102 / \mathrm{min}$, respiratory rate of $20 / \mathrm{min}$, and an oxygen saturation of $98 \%$ under $3 \mathrm{~L} / \mathrm{min}$ oxygen by reservoir mask. Coarse crackles were audible in both lungs. Skin breakdown was found on the left upper arm and the left thigh. Tenderness and loss of sensation were found from the left forearm to the wrist and from the right lower leg to the toe. She showed muscle weakness in the left forearm, the left fingers, the right thigh, and the right leg.

Laboratory findings were as follows: urinalysis showed dark brown color, $\mathrm{pH}$ of 5.0, 3+ occult blood with 5-9 red blood cells/high-power field, and $2+$ protein. Blood analysis showed hemoglobin of $13.8 \mathrm{~g} / \mathrm{dL}$, white blood cell count of $10,900 / \mu \mathrm{L}$, platelet count of $167,000 / \mu \mathrm{L}$, albumin of $3.6 \mathrm{~g} / \mathrm{dL}$, blood urea nitrogen of $22.6 \mathrm{mg} / \mathrm{dL}$, creatinine of $2.23 \mathrm{mg} / \mathrm{dL}$, creatine kinase (CK) of 57,542 $\mathrm{U} / \mathrm{L}$, myoglobin of $9,300 \mathrm{ng} / \mathrm{mL}, \mathrm{Na}$ of $136 \mathrm{mEq} / \mathrm{L}, \mathrm{K}$ of $5.4 \mathrm{mEq} / \mathrm{L}, \mathrm{Ca}$ of $7.4 \mathrm{mg} / \mathrm{dl}, \mathrm{P}$ of $5.3 \mathrm{mg} / \mathrm{dl}$, and Creactive protein of $15.43 \mathrm{mg} / \mathrm{dL}$.

Chest X-ray in the decubitus position showed a $63 \%$ cardiothoracic ratio. Plain computed tomography (CT) showed an infiltrative shadow at the left lung, suggestive of pneumonia, and a slightly atrophic kidney. Plain CT of her extremities showed swelling and low-density areas in the right quadriceps femoris muscle (Fig. 1a), indicative of muscle necrosis.

Norepinephrine was infused to maintain blood pressure immediately after admission. She received acetate Ringer's solution in $2000 \mathrm{~mL}$ boluses and maintenance hydration, but the urine output was $20 \mathrm{~mL} / \mathrm{h}$ and then further decreased. Pneumonia was treated with ampicillin sodium/sulbactam sodium. Though blood pressure was normalized, she initiated HD $11 \mathrm{~h}$ after hospitalization because of persistent oliguria. She underwent HD with a conventional polymethylmethacrylate (PMMA) high-flux dialyzer, Toray NF-1.6 $\mathrm{H}^{\circ}$ (4- to 5-h duration, blood flow 150 to $200 \mathrm{~mL} / \mathrm{min}$, dialysate flow $500 \mathrm{~mL} / \mathrm{min}$ ) at the right internal jugular vein using a venous catheter. Ultrafiltration rate during each dialysis session was no more than 8.9 $\mathrm{mL} / \mathrm{h} / \mathrm{kg}$. The total amounts of albumin in the effluent dialysate by using NF-1.6 $\mathrm{H}^{\oplus}$ (4-h duration, blood flow $270 \mathrm{~mL} / \mathrm{min}$, dialysate flow $500 \mathrm{~mL} / \mathrm{min}$ ) was reported to be about $1.8 \mathrm{~g} / \mathrm{HD}$ session [9]. She discontinued HD after 11 sessions, at which time her serum creatinine level was decreased to around the baseline value 28 days after discontinuation of HD. Her wound cured, and she was discharged 47 days after admission to a rehabilitation hospital with slight paralysis in the left arm and disuse muscle weakness. 

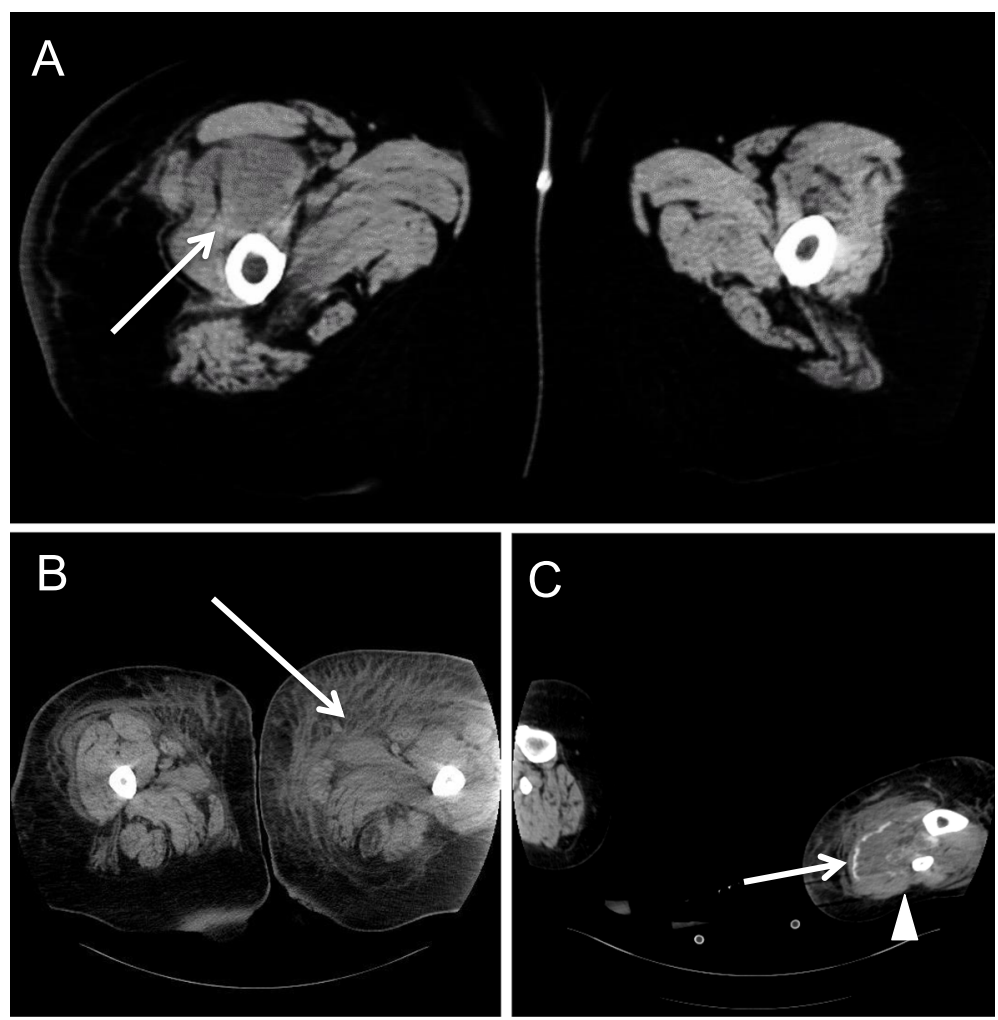

Fig. 1 Plain computed tomography (CT) images of muscle damages. a CT in case 1 shows swelling and low-density areas in the right quadriceps femoris muscle (arrow). b, c CT in case 2 shows swelling and low-density areas in the left adducent muscles (arrow) (b) and in the left gastrocnemius muscle (arrowhead) (c) and swelling and low-/high-density areas in the soleus muscle (arrow) (c)

\section{Case 2}

A 48-year-old woman fell down on the floor in the bedroom due to slipping late at night and kept lying because of damage to the left leg. She was in the supine position until she called the fire station at 4:30 pm the next day by her cell phone, which she happened to find nearby. She was transferred to our hospital. She had diabetes mellitus at the age of 43 and took metformin hydrochloride of $2250 \mathrm{mg} /$ day and tofogliflozin hydrate of 20 $\mathrm{mg} /$ day, and she took apixaban of $10 \mathrm{mg} /$ day for atrial fibrillation.

Upon admission, she complained of numbness in the left lower leg and pain in the back and the buttocks. Physical examination on admission showed alert consciousness, height of $162 \mathrm{~cm}$, body weight of $113.4 \mathrm{~kg}$, BMI of $43.2, \mathrm{~kg} / \mathrm{m}^{2}$, body temperature of $36.5^{\circ} \mathrm{C}$, blood pressure of $128 / 83 \mathrm{mmHg}$, heart rate of $113 / \mathrm{min}$, and oxygen saturation of $100 \%$ under $3 \mathrm{~L} / \mathrm{min}$ oxygen by reservoir mask. The left leg showed tenderness, redness, swelling, loss of sensation, and muscle weakness.

Laboratory findings were as follows: urinalysis showed dark brown color, $\mathrm{pH}$ of $6.5,3+$ occult blood with 5-9 red blood cells/high-power field, and 2+ protein. Blood analysis showed hemoglobin of $14.8 \mathrm{~g} / \mathrm{dL}$, white blood cell count of $30,100 / \mu \mathrm{L}$, platelet count of $468,000 / \mu \mathrm{L}$, albumin of $4.5 \mathrm{~g} / \mathrm{dL}$, blood urea nitrogen of $20.8 \mathrm{mg} / \mathrm{dL}$, creatinine of $3.13 \mathrm{mg} / \mathrm{dL}$, CK of 101,654 U/L, Na of 138 $\mathrm{mEq} / \mathrm{L}, \mathrm{K}$ of $6.2 \mathrm{mEq} / \mathrm{L}$, Ca of $6.5 \mathrm{mg} / \mathrm{dL}, \mathrm{P}$ of $6.4 \mathrm{mg} /$ $\mathrm{dL}$, and C-reactive protein of $2.02 \mathrm{mg} / \mathrm{dL}$.

The electrocardiogram showed atrial fibrillation. Intravenous calcium gluconate and glucose-insulin therapy were performed for hyperkalemia. She began to receive fluid resuscitation with 200 to $250 \mathrm{~mL}$ of isotonic saline per hour. Plain CT showed subcutaneous edema in the left lower leg and swelling and low-density areas in the left adducent muscles (Fig. 1b) and in the left gastrocnemius muscle (Fig. 1c) and swelling and low-/high-density areas in the soleus muscle (Fig. 1c), suggestive of muscle necrosis and calcification. She became hypotensive and required a norepinephrine infusion. Compartment syndrome was rejected. Creatinine was increased to 4.91 $\mathrm{mg} / \mathrm{dL}$ with oliguria despite enough hydration; thus, she required HD 4 days after admission and underwent 2 HD sessions with a conventional PMMA high-flux dialyzer, Toray NF-2.1H $1 \mathrm{H}^{\bullet}$ (4-h duration, blood flow 150 to $180 \mathrm{~mL} / \mathrm{min}$, dialysate flow $500 \mathrm{~mL} / \mathrm{min}$ ) at the right internal jugular vein using a venous catheter. Ultrafiltration rate during each dialysis session was no more than $7.5 \mathrm{~mL} / \mathrm{h} / \mathrm{kg}$. Serum creatinine was decreased to 0.55 $\mathrm{mg} / \mathrm{dL} 30$ days after discontinuation of HD. She was 
discharged 70 days after admission with slight pain in the left knee and loss of sensation and muscle weakness in the left leg. She moved to a rehabilitation hospital.

\section{Discussion}

Rhabdomyolysis is a well-recognized cause of AKI, and myoglobin, a 17,500-Da oxygen carrier, is a causative compound of rhabdomyolysis-induced AKI [1]. Recently, Stahl et al. recommend a clinical syndrome of acute muscle weakness, myalgia, and muscle swelling combined with a CK cut-off value of $>1000 \mathrm{IU} / \mathrm{L}$ or CK $>5 \times$ upper limit of normal for the standard definition of a mild rhabdomyolysis [10]. A CK level of $5000 \mathrm{U} / \mathrm{L}$ or greater associated with myoglobinuria is likely related to AKI caused by rhabdomyolysis [11]. Need of renal replacement therapy (RRT) is rarely seen with CK levels below 40,000 U/L upon admission [12]. Dehydration, acidic urine, and other comorbid conditions other than myoglobin itself are also thought to be important factors in the pathogenesis of AKI in patients with rhabdomyolysis. Our case 1 had the combination of dehydration, angiotensin receptor blocker use, and CKD, which could contribute to AKI due to reduced glomerular filtration rate. However, it is sometimes difficult to isolate the leading cause of AKI, and the term "rhabdomyolysisinduced AKI" is conventionally used under the condition.

Pressure-induced rhabdomyolysis and AKI are caused by prolonged immobilization mainly due to alcohol abuse, drug intoxication, or anesthesia for surgery [3]. It was reported that outside of surgical cases, the most common position to induce rhabdomyolysis and AKI was lateral decubitus, the lower extremities were most often involved, and $93 \%$ of patients presented with compartment syndrome in the affected muscle groups [3]. Though reported patients with pressure-induced rhabdomyolysis and AKI after falls in a state of consciousness, like our patients, are rare in the English-language literature [3, 13], there is a possibility that they were merely not reported.

Obese people are at risk for rhabdomyolysis due to trauma [4] or immobilization, especially after prolonged surgery $[5,6]$. Direct prolonged mechanical pressure on muscle tissue, resulting in muscle hypoxia and/or crush syndrome-like injury, is reported to be the main mechanism of rhabdomyolysis after prolonged surgery in obese patients [3]. In a prospective cohort study involving 49 obese patients undergoing bariatric surgery for obesity, the risk of rhabdomyolysis increased with prolonged surgery $(>4 h)$, BMI $>40 \mathrm{~kg} / \mathrm{m}^{2}$, and diabetes mellitus [5]. More recently, a multicenter, prospective study reported that after bariatric surgery, the risk of rhabdomyolysis increased when the duration of surgery was $>230 \mathrm{~min}$ [14]. Our patients had similar risks for rhabdomyolysis as people undergoing bariatric surgery, such as BMI $>40 \mathrm{~kg} / \mathrm{m}^{2}$ and immobilization of $>230 \mathrm{~min}$. Since our patients were carried into the emergency room, serum CK and creatinine were immediately measured, and the diagnosis was made. However, muscle damage after a fall at home is apt to be treated lightly because it is thought that those injuries are mild; thus, blood and urine tests might not be done in the first place in primary care institutions. As in our cases (Table 1), it has been reported that the severity of rhabdomyolysis-induced AKI did not correlate with the degree of muscle injury as reflected by serum CK activity [3], but another study found a weak linear correlation between CK and creatinine levels [15]. The number of obese people has increased worldwide, so the number of cases similar to ours must have also increased, suggesting that monitoring of serum CK and creatinine is critical not to overlook rhabdomyolysis-induced AKI in morbidly obese people.

Table 1 Clinical characteristics relevant to rhabdomyolysis-induced AKI

\begin{tabular}{lll}
\hline & Case 1 & Case 2 \\
\hline Age & 67 & 48 \\
Preexisting CKD & CKD stage G3b & No \\
Immobilization time (hour) & 12 & 16 \\
Serum CK upon admission/peak (U/L) & $57,542 / 65,550$ on HD & $101,654 / 133,234$ \\
Serum myoglobin upon admission (ng/mL) & 9,380 & Unmeasured \\
Urine myoglobin upon admission $(\mathrm{ng} / \mathrm{mL})$ & Unmeasured & $10.0 \geqq$ \\
Serum Cr upon admission/peak (mg/dL) & $2.23 / 11.35$ on HD & $3.13 / 5.95$ \\
Serum K upon admission/peak (mEq/L) & $5.4 / 5.4$ & $6.2 / 6.2$ \\
Serum corrected calcium upon admission(mg/dL) & 7.8 & 6.5 \\
Serum phosphate upon admission $(\mathrm{mg} / \mathrm{dL})$ & 5.3 & 6.4 \\
Time to HD start from hospitalization (hour) & 11 & 96 \\
Time to end from HD start (day) & 19 & 2 \\
The number of times of HD session & 11 & 2 \\
Serum creatinine at the time of the discharge $(\mathrm{mg} / \mathrm{dL})$ & 1.22 & 0.55 \\
\hline
\end{tabular}


Although the majority of patients with AKI due to rhabdomyolysis recover their renal function, the mortality in the intensive care unit has been reported to be $59 \%$ when AKI is present and $22 \%$ when it is not present [2]. Since rhabdomyolysis by crush syndrome or immobilization is usually associated with volume depletion due to the sequestration of water in injured muscles, early and aggressive fluid resuscitation using isotonic saline is recommended as a conservative therapy: it increases renal blood flow, thereby increasing the secretion of nephrotoxic compounds, such as myoglobin, maybe preventing AKI [2]. Aciduria promotes myoglobinuric-renal injury by acute precipitation of myoglobin within the kidney in animal models [16]. Therefore, alkalization of the urine is a theoretical intervention in rhabdomyolysis, but randomized controlled trials, systematic reviews, or observational studies on alkalization compared to none did not show evidence of a clinical benefit in patients with rhabdomyolysisinduced AKI [17]. Current consensus statements describe that sodium bicarbonate administration is not necessary and not superior to normal saline diuresis in increasing urine $\mathrm{pH}[17,18]$. Fluid resuscitation can sustain urine output to inhibit myoglobin concentration in renal tubules and to correct aciduria. Alkalization of the urine and mannitol use for forced diuresis are recommended under specific circumstances but have not been shown to change clinical outcomes in patients with crush syndrome $[2,12]$.

Our 2 morbidly obese women with rhabdomyolysisinduced AKI required HD for renal indication despite aggressive fluid therapy. The clinical characteristics of both cases are summarized in Table 1. Fortunately, in both cases, renal function returned to baseline after discontinuation of HD. However, severe AKI is known to be a risk for mortality and the development of CKD even after recovery [7]. At present, prophylactic RRT to remove serum myoglobin is not recommended because of no evidence of improvement of renal outcome in patients with rhabdomyolysis-induced AKI by RRT. However, various kinds of membranes, which can remove myoglobin effectively with less albumin leakage are now available in Japan and other countries [8]; thus, we need to reevaluate whether RRT can improve the renal outcome in patients with rhabdomyolysis-induced AKI. If RRT using the appropriate membrane proves to be effective, early introduction of RRT for nonrenal indications might lessen the risks for mortality and the development of CKD in patients with rhabdomyolysis-induced AKI.

\section{Minireview}

\section{Obesity and AKI}

Obesity is an independent risk factor for AKI, which, if severe enough, requires dialysis. In a cohort of 14,986 critically ill patients, each $5 \mathrm{~kg} / \mathrm{m}^{2}$ increase in BMI was associated with a $10 \%$ risk of more severe AKI [19]. There are some factors that may lead to the increased risk for AKI in obese people. Excess abdominal fat in obese patients is associated with intra-abdominal hypertension, which can predispose critically ill patients to AKI via renal venous congestion and poor arterial perfusion from decreased venous return [20]. Obesity is associated with increased levels of pro-inflammatory cytokines [21], oxidative stress [22], and endothelial dysfunction [23]. These factors are known to be associated with a development of AKI. In addition, the morbidly obese patients are more likely to present with comorbidities such as hypertension, diabetes and cardiovascular diseases [24]. Not only these co-morbidities but also obesity itself is a risk factor for CKD [25], and CKD is also an important risk factor for the development of AKI [26]. Both of our cases were prone to cause AKI because case 1 had CKD and case 2 had diabetes. Moreover, diabetes shows muscular weakness, which could partly be explained by alteration of membrane homeostasis and diabetic microangiopathy which can favor muscular ischemia [27]. Thus, besides direct prolonged mechanical pressure on muscle tissue, diabetes was also a risk for the development of rhabdomyolysis in case 2 .

Rhabdomyolysis is a widely recognized complication in statin use. Our case 1 was treated with atorvastatin. The relation of BMI to statin-associated rhabdomyolysis or muscle symptoms is controversial. Some researchers reported obesity as a risk factor [28], whereas others found that a small body frame and frailty increased the risk [29].

There were only 2 reported cases with rhabdomyolysisinduced AKI after falls in a state of consciousness, like our cases [3, 13]. A 39-year-old man did not need dialysis therapy, and another 82-year old man required a short period of HD 5 days after infusion of saline [3]. BMI was not described, but both reported cases had no previous risk factors for AKI, indicating that rhabdomyolysisinduced AKI requiring dialysis could occur after a fall on a floor in people without any risk factors for AKI except older age.

\section{RRT for rhabdomyolysis-induced AKI}

Myoglobin is a causative compound of rhabdomyolysisinduced AKI, so removal of circulating myoglobin is theoretically effective in preventing AKI in patients with rhabdomyolysis. However, the benefit of rapidly removing circulating myoglobin by RRT has not been established. Peritoneal dialysis is inadequate to remove the large solute loads in patients with rhabdomyolysis [30]. The removal of myoglobin by plasma exchange has demonstrated controversial benefits [31, 32]. We used intermittent HD in our cases for renal indication. However, there are other choices to increase removal amount of 
myoglobin, such as prolonging the time of treatment including continuous RRT (CRRT) or using hemodiafiltration (HDF) with or without albumin supplementation as well as the selection of membrane and operating condition. CRRT using conventional high-flux filters has been found to eliminate more myoglobin than standard intermittent HD [33]. Zeng et al. [34] systematically reviewed the potential benefits of CRRT compared with conventional therapy (supportive therapies and/or intermittent HD) in patients with rhabdomyolysis-induced AKI. However, there was insufficient evidence to discern any likely benefits of CRRT over conventional therapy in reducing in either mortality or preventing AKI in patients with rhabdomyolysis, and CRRT was not advocated for preventing AKI or improving renal outcome.

An increasing number of reports indicate that a highcut-off $(\mathrm{HCO})$ membrane, which can filter out molecular weights closer to that of the native kidney $(65,000 \mathrm{Da})$ and clear more myoglobin than a conventional high-flux membrane (MW cut-off of 10,000-20,000 Da), is beneficial for rhabdomyolysis-induced AKI [33, 35-40]. A case series of patients with severe rhabdomyolysis demonstrated that continuous veno-venous HD (CVVHD) and sustained low-efficiency daily dialysis with $\mathrm{HCO}$ membranes allowed a considerable removal of myoglobin, $60 \%$ after $2 \mathrm{~h}$ and $70 \%$ after $4 \mathrm{~h}$ in the latter condition [38]. In 2 case reports, patients with rhabdomyolysis showed a decline in serum myoglobin level by $50 \%$ after $4 \mathrm{~h}$ or $50-55 \%$ after $1 \mathrm{~h}$ and by $61-71 \%$ after $3 \mathrm{~h}$ using HCO intermittent HD, respectively $[39,40]$. In a prospective, randomized, single-blindedsingle-center trial, 60 critically ill patients with AKI and established indications for RRT were randomized 1:1 into two groups. The trial found a significantly better elimination of myoglobin in the intervention group with CVVHD using the HCO membrane than with CVVHD using the conventional high-flux membrane. Despite this theoretical advantage, the $\mathrm{HCO}$ membrane still suffers from a loss of unacceptable amounts of albumin (MW 66,000 Da) and other essential proteins and a high price [41].

The medium cut-off (MCO) membrane exhibits highly selective permeability and have both high-quality and good performance for dialysis treatment, showing the removal of large to middle-sized molecules, up to MW 45, $000 \mathrm{Da}$, including the $17,500-\mathrm{Da}$ myoglobin, like HCO membranes, and the low removal of albumin, like most advanced high-flux membranes [42]. It can achieve the same removal performance as that achieved by HDF [43]. On the other hand, the solute removal characteristics of the MCO membrane are considered to be almost the same as those of the type IIa dialyzer (albumin sieving coefficient $<0.03, \beta 2 \mathrm{MG}$ clearance $>70 \mathrm{~mL} / \mathrm{min}$ ) in the current Japanese classification. It was reported that MCO dialyzer is superior to conventional HD with standard high-flux dialyzers in the removal of middle and larger middle molecules and it is not inferior to online-HDF [44]. Mean reduction rate (RR) of myoglobin using high-flux HD with FX80 dialyzer (Fresenius Medical Care, Bad Homburg, Germany), HD with MCO Theranova dialyzer (Gambro Dialysatoren, Hechingen, Germany, a subsidiary of Baxter International), and online-HDF using FX 1000 dialyzer (Fresenius Medical Care) was $34.3 \%, 62.5 \%$, and $72.4 \%$, respectively [44]. We measured serum myoglobin before and after HD session in case 1 , and $R R$ of myoglobin was calculated to be $61.6 \%$. RR of myoglobin using HD with the PMMA dialyzer NF-H 1.6 in our case 1 was comparable to that using HD with MCO dialyzer.

The PMMA membrane belongs to the type $\mathrm{S}$ dialyzer with special biocompatibility in Japanese classification and has an absorbed ability of several kinds of proteins including interleukin-6 [45, 46]. Obese people have higher baseline levels of pro-inflammatory mediators such as interleukin-6 which may increase the risk for the developing AKI [47]. Thus, the treatment with the PMMA dialyzer NF-H can be considered to have a preventive effect on rhabdomyolysis-induced AKI in morbidly obese people in terms of sufficient removal of myoglobin and the absorbed ability of interleikin-6.

Rapidly removing circulating myoglobin just after rhabdomyolysis may be essential for preventing AKI. However, to achieve elimination of serum myoglobin to a nontoxic level, we need to know how we should decide to start RRT and also to discontinue RRT, especially when myoglobin release is prolonged significantly. CK, myoglobin, and urine dipstick are among the most studied predictive factors for AKI following rhabdomyolysis. Although CK peak is a late severity marker of rhabdomyolysis, CK level of $5000 \mathrm{U} / \mathrm{L}$ or greater, which is likely related to AKI caused by rhabdomyolysis [11], is one of the indices to start preventive dialysis treatment. Serum myoglobin level peaks 8 to $12 \mathrm{~h}$ after muscle damage, followed by the serum CK peak [48]. Serum myoglobin was reported to show unpredictable metabolism, partly in the kidney but mainly outside the kidney, probably in the liver or spleen $[48,49]$. Serum myoglobin has a rapid metabolism and disappears from circulation within $24 \mathrm{~h}$ when there is no further release of myoglobin [48], indicating that the serum myoglobin level is extremely dependent on the timing of measurement. Therefore, serum myoglobin levels are not reliable for the diagnosis or management of rhabdomyolysis. Like urine myoglobin level upon admission in our case 2 in Table 1, urine myoglobin is not a sensitive test for rhabdomyolysis, nor is it specific for the development of AKI; therefore, it is not necessary for routine testing $[50,51]$. The presence of increasing amounts of blood by urinalysis progressively raises the probability of myoglobinuria, especially 
in the absence of hematuria. This predictive information may be useful for the initial assessment of patients with rhabdomyolysis and perhaps for detection of patients at risk for AKI following rhabdomyolysis and monitoring urine myoglobin clearance [52]. Thus, urinalysis might be applicable for another index to start preventive dialysis treatment. However, there are limited data about the degree of positive urine dipstick for blood and cut-off point of red blood cells per high power field related to AKI. Moreover, it is not known how clinicians should evaluate the patients with pre-existing hematuria like in our case 2 with preexisting CKD.

\section{Conclusions}

We should be aware of the importance of monitoring rhabdomyolysis so as not to overlook it in morbidly obese people who are at risk for rhabdomyolysis after prolonged immobilization. High-quality randomized controlled trials are needed to evaluate whether RRT using the appropriate membrane to effectively remove myoglobin including the PMMA membrane could become the first line of preventive measures for rhabdomyolysis-induced AKI.

\section{Abbreviations}

AKI: Acute kidney injury; BMI: Body mass index; CK: Creatine kinase; HCO: High cut-off; HD: Hemodialysis; HDF: Hemodiafiltration; CKD: Chronic kidney disease; CRRT: Continuous renal replacement therapy; CT: Computed tomography; CWHD: Continuous veno-venous hemodialysis; MCO: Medium cut-off; MW: Molecular weight; PMMA: Polymethylmethacrylate;

RR: Reduction rate; RRT: Renal replacement therapy

\section{Acknowledgements}

Not applicable

\section{Authors' contributions}

$\mathrm{KS}, \mathrm{HO}, \mathrm{RT}, \mathrm{YH}, \mathrm{MU}, \mathrm{YT}, \mathrm{YN}, \mathrm{SA}, \mathrm{MN}, \mathrm{SA}, \mathrm{OY}$, and $\mathrm{YT}$ analyzed and interpreted the patient data. YF, SS, and SU drafted the manuscript, and YF is responsible for the final version of the manuscript. All authors read and approved the final manuscript.

\section{Funding}

The authors declare that there is no funding related to this manuscript.

\section{Availability of data and materials}

All data analyzed during this study are included in this published article.

\section{Ethics approval and consent to participate}

The case report was written in compliance with the Declaration of Helsinki.

\section{Consent for publication}

Written informed consent was obtained from the patient for the publication of this case report.

\section{Competing interests}

The authors declare that they have no competing interests.

Received: 24 December 2019 Accepted: 24 June 2020

Published online: 08 July 2020

\section{References}

1. Petejova N, Martinek A. Acute kidney injury due to rhabdomyolysis and renal replacement therapy: a critical review. Crit Care. 2014;18:224.
2. Bosch X, Poch E, Grau JM. Rhabdomyolysis and acute kidney injury. N Engl J Med. 2009;361:62-72.

3. Szewczyk D, Ovadia P, Abdullah F, Rabinovici R. Pressure-induced rhabdomyolysis and acute renal failure. J Trauma. 1998:44:384-8.

4. Chan JL, Imai T, Barmparas G, Lee JB, Lamb AW, Melo N, et al. Rhabdomyolysis in obese trauma patients. Am Surg. 2014;80:1012-7.

5. Lagandré S, Arnalsteen L, Vallet B, Robin E, Jany T, Onraed B, et al. Predictive factors for rhabdomyolysis after bariatric surgery. Obes Surg. 2006;16:136570

6. Lebuffe G, Andrieu G, Wierre F, Gorski K, Sanders V, Chalons N, et al. Anesthesia in the obese. J Visc Surg. 2010;147(Suppl 5):e11-9.

7. Bucaloiu ID, Kirchner HL, Norfolk ER, Hartle JE 2nd, Perkins RM. Increased risk of death and de novo chronic kidney disease following reversible acute kidney injury. Kidney Int. 2012;81:477-85.

8. Florens $N$, Juillard L. Expanded haemodialysis: news from the field. Nephrol Dial Transplant. 2018;33(Suppl 3):iii48-52.

9. Masakane I, Esashi S, Yoshida A, Chida T, Fujieda H. Ueno Y, et al. Renal replacement therapy. 2017:3:32.

10. Stahl K, Rastelli E, Schoser B. A systematic review on the definition of rhabdomyolysis. J Neurol. 2019. https://doi.org/10.1007/s00415-019-09185-4 [Epub ahead of print].

11. Brown C, Rhee P, Chan L, Evans K, Demetriades D, Velmahos GC. Preventing renal failure in patients with rhabdomyolysis: do bicarbonate and mannitol make a difference? J Trauma. 2004;56:1191-6.

12. de Meijer AR, Fikkers BG, de Keijzer MH, van Engelen BG, Drenth JP. Serum creatine kinase as predictor of clinical course in rhabdomyolysis: a 5-year intensive care survey. Intensive Care Med. 2003;29:1121-5.

13. Ratcliffe PJ, Berman P, Griffiths RA. Pressure induced rhabdomyolysis complicating an undiscovered fall. Age Ageing. 1983;12:245-8.

14. Tolone S, Pilone V, Musella M, Rossetti G, Milone M, Fei L, et al Rhabdomyolysis after bariatric surgery: a multicenter, prospective study on incidence, risk factors, and therapeutic strategy in a cohort from South Italy. Surg Obes Relat Dis. 2016;12:384-90.

15. Melli G, Chaudhry V, Cornblath DR. Rhabdomyolysis: an evaluation of 475 hospitalized patients. Medicine (Baltimore). 2005;84:377-85.

16. Zager RA. Studies of mechanisms and protective maneuvers in myoglobinuric acute renal injury. Lab Invest. 1989;60:619-29.

17. Michelsen J, Cordtz J, Liboriussen L, Behzadi MT, Ibsen M, Damholt MB, et al. Prevention of rhabdomyolysis-induced acute kidney injury - a DASAIM/ DSIT clinical practice guideline. Acta Anaesthesiol Scand. 2019;63:576-86.

18. Brochard L, Abroug F, Brenner M, Broccard AF, Danner RL, Ferrer M, et al. ATS/ERS/ESICM/SCCM/SRLF Ad Hoc Committee on Acute Renal Failure. An Official ATS/ERS/ESICM/SCCM/SRLF Statement: Prevention and Management of Acute Renal Failure in the ICU Patient: an international consensus conference in intensive care medicine. Am J Respir Crit Care Med. 2010;181: $1128-55$.

19. Danziger J, Chen KP, Lee J, Feng M, Mark RG, Celi LA, et al. Obesity, acute kidney injury, and mortality in critical illness. Crit Care Med. 2016;44:328-34.

20. Shashaty MG, Kalkan E, Bellamy SL, Reilly JP, Holena DN, Cummins K, et al. Computed tomography-defined abdominal adiposity is associated with acute kidney injury in critically ill trauma patients. Crit Care Med. 2014;42: 1619-28.

21. Alexopoulos N, Katritsis D, Raggi P. Visceral adipose tissue as a source of inflammation and promoter of atherosclerosis. Atherosclerosis. 2014;233: 104-12.

22. Billings FT 4th, Pretorius M, Schildcrout JS, Mercaldo ND, Byrne JG, Ikizler TA, Brown NJ. Obesity and oxidative stress predict AKI after cardiac surgery. J Am Soc Nephrol. 2012;23:1221-8

23. Romero-Corral A, Sert-Kuniyoshi FH, Sierra-Johnson J, Orban M, Gami A Davison D, et al. Modest visceral fat gain causes endothelial dysfunction in healthy humans. J Am Coll Cardiol. 2010:56:662-6.

24. Sowers JR. Obesity as a cardiovascular risk factor. Am J Med. 2003;115(Suppl 8A):37S-41S.

25. Yun HR, Kim H, Park JT, Chang TI, Yoo TH, Kang SW, et al. Korean Cohort Study for Outcomes in Patients With Chronic Kidney Disease (KNOW-CKD) Investigators. Obesity, metabolic abnormality, and progression of CKD. Am J Kidney Dis. 2018;72:400-10.

26. He L, Wei Q, Liu J, Yi M, Liu Y, Liu H, et al. AKI on CKD: heightened injury, suppressed repair, and the underlying mechanisms. Kidney Int. 2017;92: 1071-83. 
27. Ziser A, Friedhoff RJ, Rose SH. Prone position: visceral hypoperfusion and rhabdomyolysis. Anesth Analg. 1996 Feb;82(2):412-5.

28. Ochs-Balcom HM, Nguyen LM, Ma C, Isackson PJ, Luzum JA, Kitzmiller JP, et al. Clinical features related to statin-associated muscle symptoms. Muscle Nerve. 2019;59:537-43.

29. Antons KA, Williams CD, Baker SK, Phillips PS. Clinical perspectives of statininduced rhabdomyolysis. Am J Med. 2006;119:400-9.

30. Nolph KD, Whitcomb ME, Schrier RW. Mechanisms for inefficient peritoneal dialysis in acute renal failure associated with heat stress and exercise. Ann Intern Med. 1969;71:317-36.

31. Szpirt WM. Plasmapheresis is not justified in treatment of rhabdomyolysis and acute renal failure. J Cardiovasc Surg (Torino). 1997;38:557.

32. Swaroop R, Zabaneh R, Parimoo N. Plasmapheresis in a patient with rhabdomyolysis: a case report. Cases J. 2009;2:8138.

33. Sorrentino SA, Kielstein JT, Lukasz A, Sorrentino JN, Gohrbandt B, Haller H, et al. High permeability dialysis membrane allows effective removal of myoglobin in acute kidney injury resulting from rhabdomyolysis. Crit Care Med. 2011;39:184-6.

34. Zeng X, Zhang L, Wu T, Fu P. Continuous renal replacement therapy (CRRT) for rhabdomyolysis. Cochrane Database Syst Rev. 2014;6:CD008566.

35. Naka T, Jones D, Baldwin I, Fealy N, Bates S, Goehl H, Morgera S, Neumayer $\mathrm{HH}$, Bellomo R. Myoglobin clearance by super high-flux hemofiltration in a case of severe rhabdomyolysis: a case report. Crit Care. 2005;9:R90-5.

36. Premru V, Kovač J, Buturović-Ponikvar J, Ponikvar R. High cut-off membrane hemodiafiltration in myoglobinuric acute renal failure: a case series. Ther Apher Dial. 2011;15:287-91

37. Wu B, Gong D, Ji D, Xu B, Liu Z. Clearance of myoglobin by high cutoff continuous veno-venous hemodialysis in a patient with rhabdomyolysis: a case report. Hemodial Int. 2015;19:135-40.

38. Heyne N, Guthoff M, Krieger J, Haap M, Häring HU. High cut-off renal replacement therapy for removal of myoglobin in severe rhabdomyolysis and acute kidney injury: a case series. Nephron Clin Pract. 2012;121:c159-64.

39. Albert C, Haase M, Bellomo R, Mertens PR. High cut-off and high-flux membrane haemodialysis in a patient with rhabdomyolysis-associated acute kidney injury. Crit Care Resusc. 2012;14:159-62.

40. Goubella A, Gankam-Kengne F, Baudoux T, Fagnoul D, Husson C, Delforge ML, Broeders N, Nortier JL. Severe myoglobinuric acute kidney injury in a kidney recipient: rapid recovery after hemodialysis with the super high-flux membrane Theralite ${ }^{\oplus}$. Clin Nephrol. 2017;88:359-63.

41. Weidhase L, Haussig E, Haussig S, Kaiser T, de Fallois J, Petros S. Middle molecule clearance with high cut-off dialyzer versus high-flux dialyzer using continuous veno-venous hemodialysis with regional citrate anticoagulation: a prospective randomized controlled trial. PLoS One. 2019;14:e0215823.

42. Zweigart C, Boschetti-de-Fierro A, Hulko M, Nilsson LG, Beck W, Storr M, Krause B. Medium cut-off membranes - closer to the natural kidney removal function. Int J Artif Organs. 2017;40:328-34.

43. Kirsch AH, Lyko R, Nilsson LG, Beck W, Amdahl M, Lechner P, Schneider A, Wanner C, Rosenkranz AR, Krieter DH. Performance of hemodialysis with novel medium cut-off dialyzers. Nephrol Dial Transplant. 2017:32:165-72.

44. García-Prieto A, Vega A, Linares T, Abad S, Macías N, Aragoncillo I, et al. Evaluation of the efficacy of a medium cut-off dialyser and comparison with other high-flux dialysers in conventional haemodialysis and online haemodiafiltration. Clin Kidney J. 2018:11:742-6.

45. Hirayama Y, Oda S, Wakabayashi K, Sadahiro T, Nakamura M, Watanabe E, et al. Comparison of interleukin-6 removal properties among hemofilters consisting of varying membrane materials and surface areas: an in vitro study. Blood Purif. 2011;31(1-3):18-25.

46. Oshihara W, Fujieda H, Ueno Y. A new poly(methyl methacrylate) membrane dialyzer, NF, with adsorptive and antithrombotic properties. Contrib Nephrol. 2017;189:230-6.

47. Eder K, Baffy N, Falus A, Fulop AK. The major inflammatory mediator interleukin-6 and obesity. Inflamm Res. 2009;58:727-36.

48. Parekh R, Care DA, Tainter CR. Rhabdomyolysis: advances in diagnosis and treatment. Emerg Med Pract. 2012;14:1-15.

49. Wakabayashi Y, Kikuno T, Ohwada T, Kikawada R. Rapid fall in blood myoglobin in massive rhabdomyolysis and acute renal failure. Intensive Care Med. 1994;20:109-12.

50. Zimmerman Jl, Shen MC. Rhabdomyolysis. Chest. 2013:144:1058-65.

51. Rodríguez-Capote K, Balion CM, Hill SA, Cleve R, Yang L, El Sharif A. Utility of urine myoglobin for the prediction of acute renal failure in patients with suspected rhabdomyolysis: a systematic review. Clin Chem. 2009;55:2190-7.
52. Safari S, Yousefifard M, Hashemi B, Baratloo A, Forouzanfar MM, Rahmati F, et al. The role of scoring systems and urine dipstick in prediction of rhabdomyolysis-induced acute kidney injury: a systematic review. Iran J Kidney Dis. 2016;10:101-6.

\section{Publisher's Note}

Springer Nature remains neutral with regard to jurisdictional claims in published maps and institutional affiliations.
Ready to submit your research? Choose BMC and benefit from:

- fast, convenient online submission

- thorough peer review by experienced researchers in your field

- rapid publication on acceptance

- support for research data, including large and complex data types

- gold Open Access which fosters wider collaboration and increased citations

- maximum visibility for your research: over $100 \mathrm{M}$ website views per year

At BMC, research is always in progress.

Learn more biomedcentral.com/submissions 